POS PROCEEDINGS

\title{
Advances on micro-RWELL gaseous detector
}

\section{G. Morello*}

Laboratori Nazionali di Frascati - INFN, Frascati, Italy

E-mail: morello@lnf.infn.it

\section{G. Bencivenni, L. Benussi, P. De Simone, G. Felici, M. Gatta, M. Poli Lener}

Laboratori Nazionali di Frascati - INFN, Italy

\section{R. De Oliveira}

CERN, $\mathrm{CH}$

\section{A. Ochi}

University of Kobe, Japan

\section{Borgonovi, P. Giacomelli}

INFN - Sez. Bologna, Italy

\section{A. Ranieri, V. Valentino}

INFN - Sez. Bari, Italy

\section{Ressegotti, I. Vai}

INFN - Sez. Pavia, Italy

The R\&D on the micro-Resistive-WELL ( $\mu$-RWELL) detector technology aims in developing a new scalable, compact, spark-protected, single amplification stage Micro-Pattern Gas Detectors (MPGD) for large area HEP applications as tracking and calorimeter device as well as for industrial and medical applications as X-ray and neutron imaging gas pixel detector. The novel microstructure, exploiting several solutions and improvements achieved in the last years for MPGDs, in particular for GEMs and Micromegas, is an extremely simple detector allowing an easy engineering with consequent technological transfer toward the photolithography industry. Large area detectors (up $1 \times 2 \mathrm{~m}^{2}$ ) can be realized splicing $\mu$-RWELL_PCB tiles of smaller size (about $0.5 \times 1 \mathrm{~m}^{2}$ - typical PCB industrial size). The detector, composed by few basic elements such as the readout-PCB embedded with the amplification stage (through the resistive layer) and the cathode defining the gas drift-conversion gap has been largely characterized on test bench with $\mathrm{X}$-ray and with beam test.

55th International Winter Meeting on Nuclear Physics

23-27 January, 2017

Bormio, Italy

\footnotetext{
* Speaker.
} 


\section{Introduction}

Every Micro Pattern Gaseous Detector (MPGD) suffers spark occurrence due to the micrometric distance between the electrodes of the amplification stage. The introduction of a thin resistive layer coupled with the readout [1] stands for a deep innovation in this field, providing a protection to the front-end electronics once the discharge occurs [2]. The detector described in this work enters the subset of the resistive MPGD, combining its stability under heavy irradiation with a very easy assembly procedure: the micro-Resistive WELL ( $\mu$-RWELL) [3]. Actually the detector is composed of just two active elements: an amplification stage ( $\mu$-RWELL_PCB) and a cathode defining the drift/conversion gas gap (fig. 1). The limited number of elements, which do not undergo time-consuming processes after their delivery, suggests an as well restricted working time for the mechanical sealing with a frame, obviously equipped with the proper o-ring.

\section{Detector architecture}

The most complex part of this detector is the $\mu$-RWELL_PCB: a stack of a GEM-like patterned foil glued onto a standard readout PCB. The GEM-like foil [4] is a $50 \mu \mathrm{m}$ thick kapton sheet clad on one face with copper (5 micron) and sputtered on the other surface with few hundreds nanometer of Diamond Like Carbon (DLC) [5]. The $\mu$-RWELL-PCB is then chemically etched to pattern the holes that act as charge amplification channels exploiting the standard photolithographic technique used to produce large area foils [6] and well consolidated after many years of GEM foils production.

The DLC operating principle is quite simple and can be summarized in few step: when the amplification charge transits from avalanche to streamer mode, the DLC charges-up consequently reducing the amplification field and then quenching the discharge. The charge collected on the resistive layer flows towards the ground with a characteristic time $\tau$ [7], dependent on the surface resistivity $\rho_{s}$ and on the capacitive coupling with the readout. This charge depletion time limits the capability of the detector to stand very high radiation fluxes; this feature can be improved introducing a second resistive sheet connected to the first through a suitable density of conductive vias. Three prototypes have been realized, two implementing the single layer (SL) scheme (declared $\rho_{s}=80,880 \mathrm{M} \Omega / \square$ ) and a third with the double layer (DL) scheme (declared $\rho_{s}=12 \mathrm{M} \Omega / \square$ ). They have been first characterized at LNF with $5.9 \mathrm{keV}$ X-rays performing, in current mode, measurements of the gain and of the rate capability under local irradiation.

\section{Detector characterization}

The gain as a function of the voltage applied to the amplification stage is reported in fig. 2: the detectors, flushed with $\mathrm{Ar}_{10} \mathrm{iC}_{4} \mathrm{H}_{10}$ 90:10, have safely reached gain values over $10^{4}$. In this case the gain is parametrized as $G_{0}=e^{\alpha \Delta V+\beta}$.

The rate capability has been then measured operating the detectors at the gain G 4000. The Xrays gun (Philips PW2217/20) has been equipped with a cylindrical collimator with radius 1.25 $\mathrm{mm}$. The variation of the gain as a function of the photon conversion rate is shown in fig. 3 . The 


\section{Drift cathode PCB}

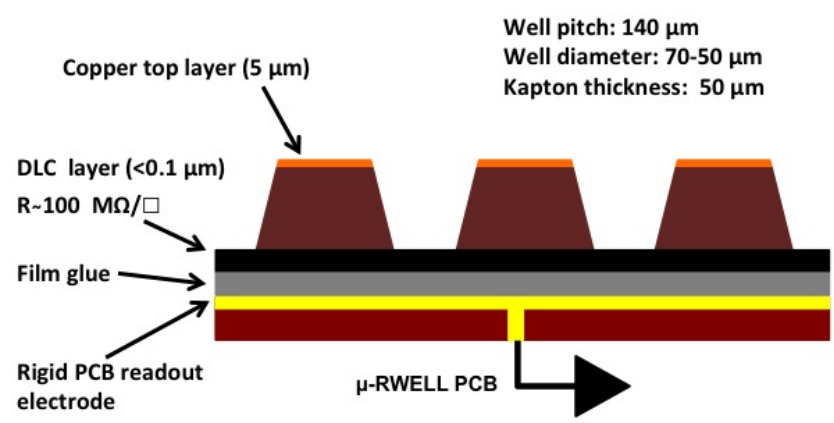

Figure 1: Sketch of the $\mu$-Resistive WELL.

points are fitted with the following function [3]

$$
\frac{G}{G_{0}}=\frac{-1+\sqrt{4 p_{0} \Phi}}{2 p_{0} \Phi}
$$

where $\mathrm{G}_{0}$ is the nominal gain and $p_{0}$ is the fit parameter depending on the primary ionization and on the resistivity. Reverting the function it is possible to compute the particle flux $\Phi$ (rate per unit area) corresponding to a given gain drop. In this work we define rate capability the flux causing a gain drop of $3 \%$.

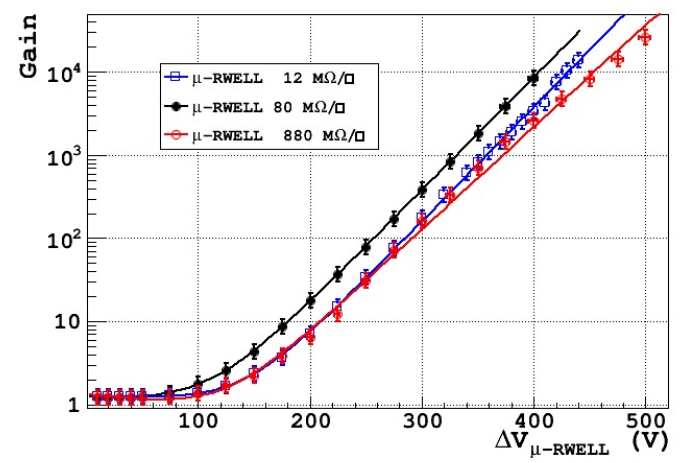

Figure 2: Measured gain for the three detectors.

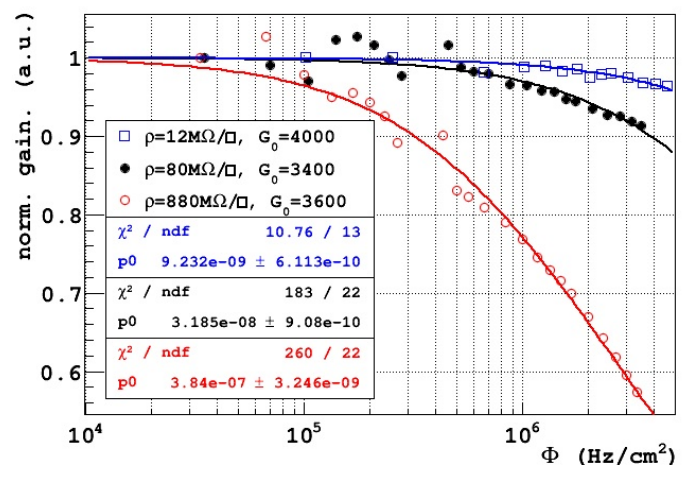

Figure 3: Normalized gain for $12 \mathrm{M} \Omega / \square$ (green triangles), $80 \mathrm{M} \Omega / \square$ (black full circles) and $880 \mathrm{M} \Omega / \square$ (red empty circles) 


\section{Test beam results}

The three detectors have been tested at the SPS-H4 North Area at CERN Prévessin with a 150 $\mathrm{GeV}$ muon beam inside the GOLIATH magnet. The devices have been operated with $\mathrm{Ar}_{\mathrm{iC}} \mathrm{H}_{10}$ 90:10 and equipped with APV25 front-end electronics [8] handled by a Scalable Readout System (SRS). For all the detectors the readout is segmented with $400 \mu \mathrm{m}$ pitch strips, performing the analysis with the Charge Centroid (CC) technique. Four external GEM trackers, provided by BESIII-Italy collaboration, have composed the telescope of the system, triggered by two scintillators pairs upstream and downstream and out of the magnetic field. The single layer prototypes exhibit a tracking efficiency over $98 \%$, reaching a plateau at gain $\mathrm{G} \sim 4000$. The double layer prototype reaches a $99 \%$ plateau for gains over $10^{4}$ : this difference can be ascribed to its low resistivity. In this case indeed the charge spread on the resistive surface is quite fast and a larger number of strips can be fired, thus requiring a higher gain to reach the full detection efficiency. A confirmation of this effect is clearly visible in fig. 5, where the number of fired strips for the 12 $\mathrm{M} \Omega / \square$ prototype is much larger than for the other detectors. On the other hand, if the resistivity is too high, the charge spread is much slower than the FEE time window, so that the number of fired strips is limited to one. A good tuning of the surface resistivity is then mandatory according to the detector application, as shown in fig. 6. A comparison has been eventually done operating the detectors at a gain $\mathrm{G} \sim 10^{4}$ and reporting the residuals width $\left(\sigma_{\text {res }}\right)$ and the strips cluster size as a function of the resistivity. At high resistivity the number of fired strips is so limited that the CC method is no longer effective and the spatial resolution reduces to pitch $/ \sqrt{12}$.

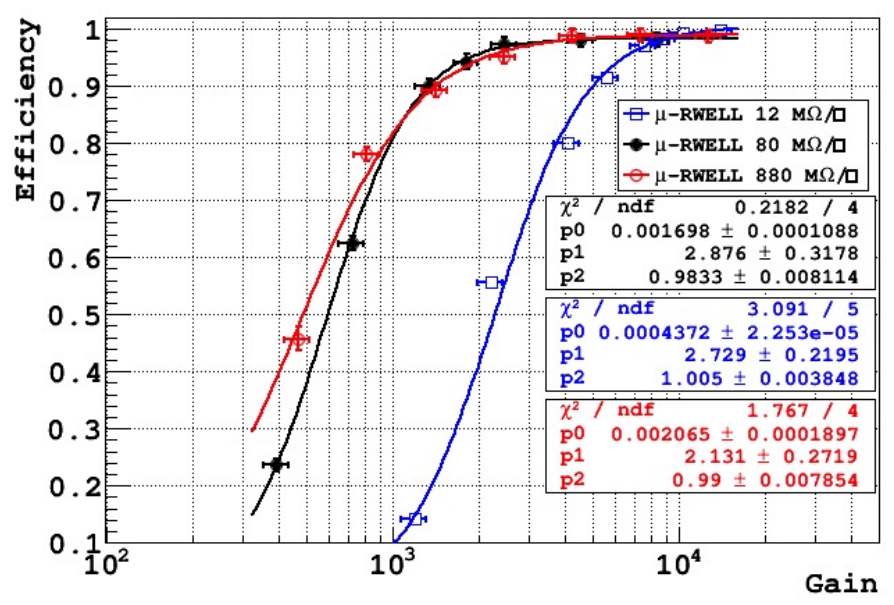

Figure 4: Tracking efficiency as a function of the gain for the three detectors.

\section{Large area $\mu$-RWELL}

In the framework of the CMS-phase2 muon upgrade the LNF-DDG group, in collaboration with the CMS groups present at LNF, INFN-Bologna and INFN-Bari, is developing a very large size $\mu$-RWELL (fig. 7) proposed for the installation in the CMS GE2/1 tracking wheels (fig. 8). The 


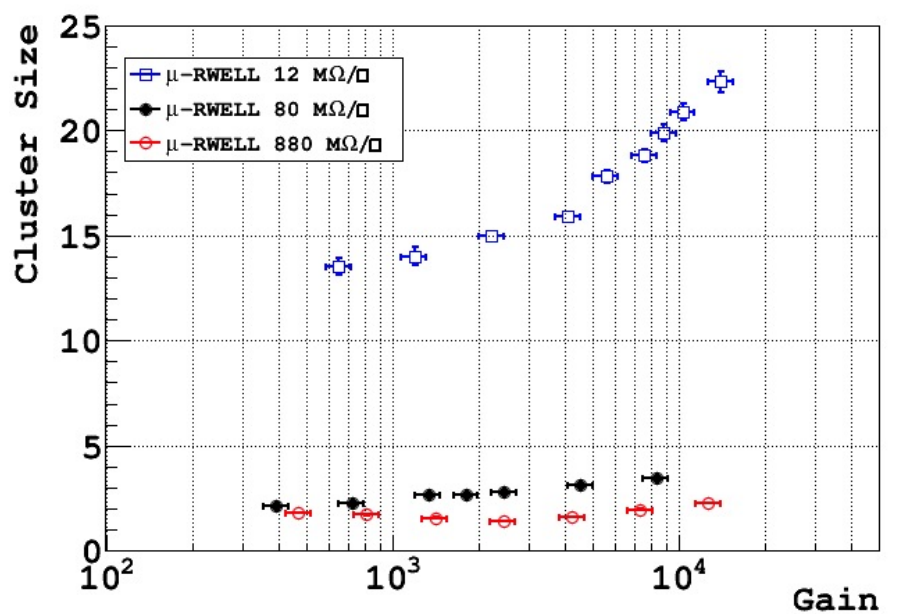

Figure 5: Strip cluster size (average number of contiguous fired strip per track) as a function of the gain for the three detectors.

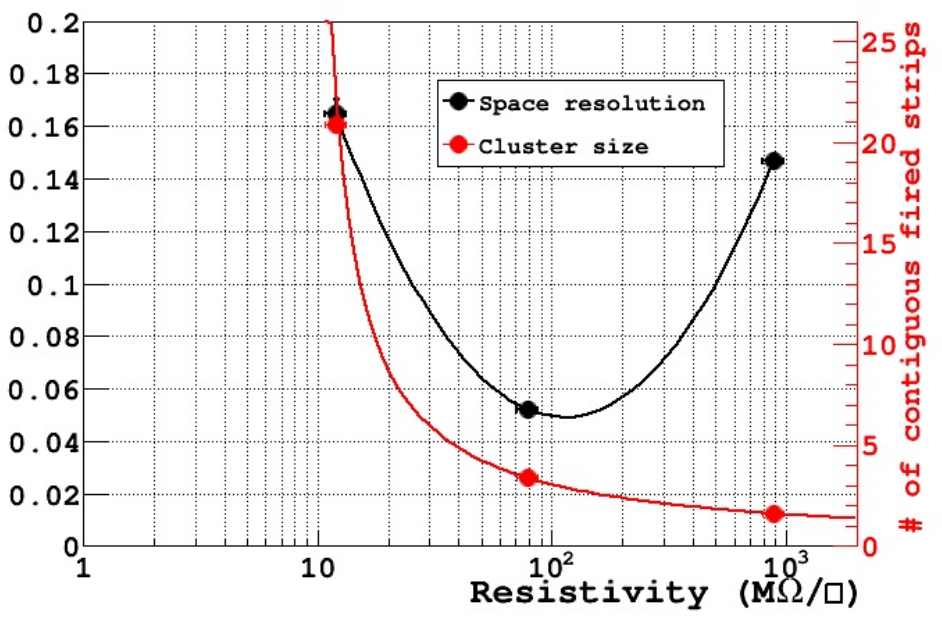

Figure 6: Residuals sigma and strip cluster size as a function of the DLC resistivity.

two wheels covers the pseudorapidity range $1.6<|\eta|<2.4$ and they are composed of 36 chambers each covering $20^{\circ}$ in the transverse plane. For this reason the chamber is trapezoidal shaped with the dimensions reported in fig. 7. The project passes by an intermediate step: the construction of GE1/1-like $\mu$-RWELL, approximately 4 times smaller than the final prototype, so far representing the largest micro-Resistive WELL ever built and with the active area segmented in 8 sectors. Two of them, covering the same pseudorapidity range, have been tested at the CERN SPS H8 test beam area, operating the detector with $\mathrm{Ar}: \mathrm{CO}_{2}: \mathrm{CF}_{4}$ 45:15:40 and readout with VFAT2 Front-end electronics, in order to measure the time performance of the detector, with or-ed strips. The same test beam involved other two $\mu$-RWELL Double Layer prototypes $\left(\rho_{s} \sim 40 \mathrm{M} \Omega / \square\right)$. The telescope 
has been composed of two GEM trackers, triggered by three scintillators stations.

The analysis is based on the selection of time coincidences of the events. Fig. 9 is an example reporting graphically the selections applied to TDC distributions relative to the external trackers and to the detector under examination. Such cuts reduce the $\mu$-RWELLs TDC spectra to a gaussian-like distribution (fig. 10) that can be easily fitted. The $\sigma_{T D C}$ must be deconvoluted by the contribution of the VFAT2 electronics in order to compute the time resolution of the detector, according to the formula [10]:

$$
\sigma_{t}=\sqrt{\sigma_{T D C}^{2}-\left(\frac{25 \mathrm{~ns}}{\sqrt{12}}\right)^{2}}
$$

The so-obtained $\sigma_{t}$ have been measured as a function of the detector gain and they are shown in fig. 11. A clear saturation is visible at higher gain, when the $\sigma_{t}$ approaches the value of $5.7 \mathrm{~ns}$. It is natural to compare this value to the one measured with triple-GEM [11]: $4.5 \mathrm{~ns}$ equipping the detector with VTX chip and constant fraction discriminator (same gas mixture). The possibility to change the beam section dimensions has been exploited to integrate the rate capability points with lower fluxes, estimated using the counters and the MWPC present in the area which provide the beam profile in both dimensions. The detectors have been operated at different gain: $10^{4}$ for the DL, 6000 and 4000 for the two sectors of the large area and the relative variation of these values has been studied as a function of the different flux (fig. 12).

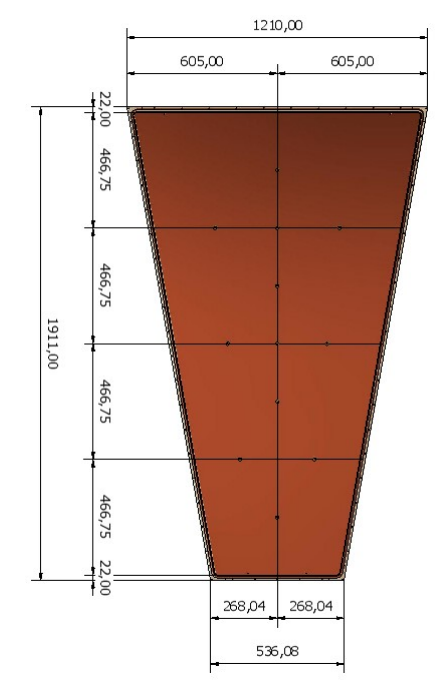

Figure 7: Dimensions of the GE2/1-like $\mu$-RWELL.

\section{Conclusions}

The $\mu$-RWELL is a thin, simple and robust MPGD for very large area applications in harsh environment. The detector exhibits a gas gain up to and above $10^{4}$ with $\mathrm{Ar}: \mathrm{i}-\mathrm{C}_{4} \mathrm{H}_{10}$ 90:10 gas mixture. The rate capability for the single-resistive layout measured with a $\sim 3 \times 3 \mathrm{~cm}^{2}$ (FWHM) pion beam is larger than $35 \mathrm{kHz} / \mathrm{cm}^{2}$, while for the double-resistive layout a rate capability better 


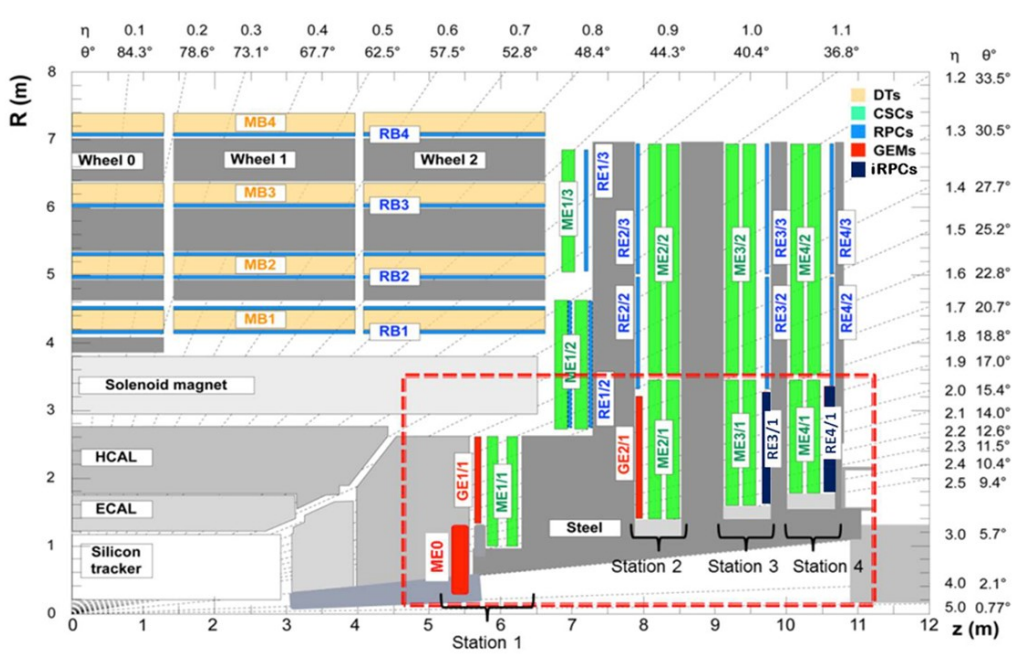

Figure 8: Section of the CMS apparatus with underlined the GE1/1 and GE2/1 station.

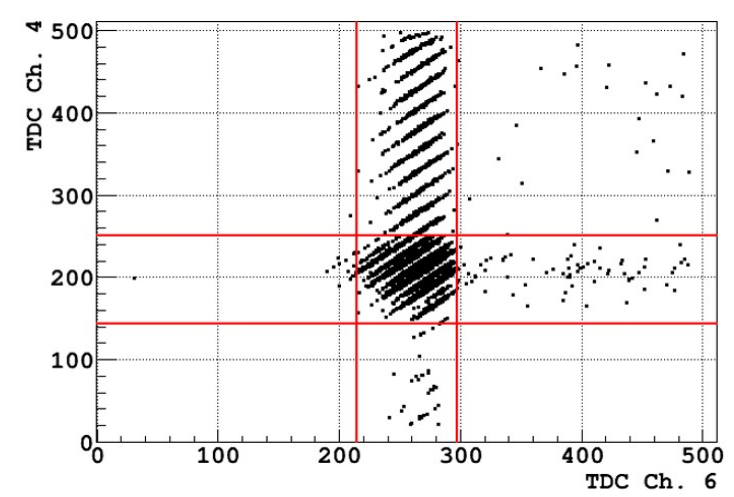

Figure 9: Distribution of the TDC events from the first external tracker correlated with the events collected by the large area $\mu$-RWELL.

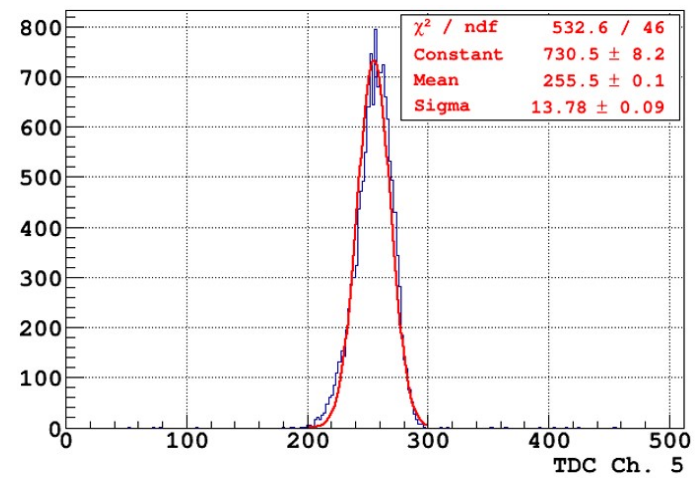

Figure 10: Example of TDC distribution for large $\mu$-RWELL after TDC selection cuts.

than $1 \mathrm{MHz} / \mathrm{cm}^{2}$ has been achieved with X-rays. A time resolution down to $5.7 \mathrm{~ns}$ has been obtained for small as well as large area detector prototypes. 


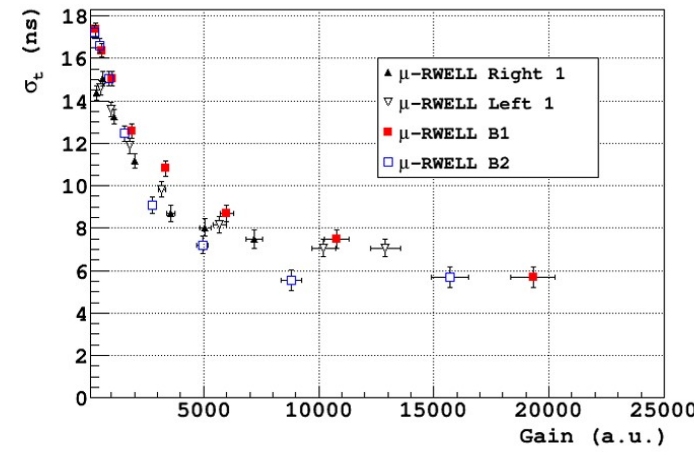

Figure 11: Time resolution as a function of the detector gain.

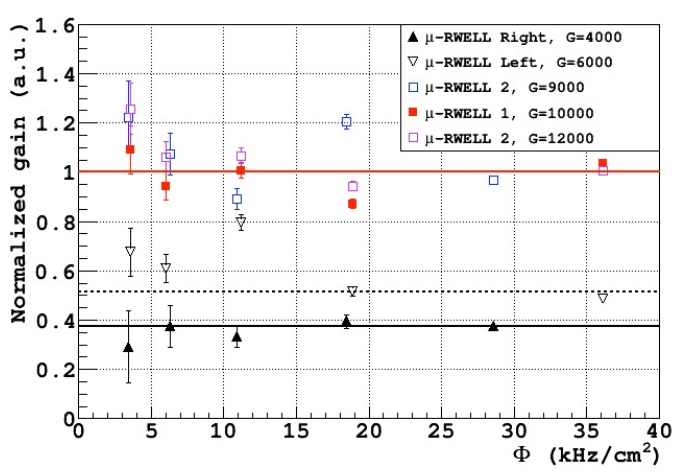

Figure 12: Gain variation as a function of the radiation fluence for the two sectors of the large area $\mu$ RWELL (triangles) and for the small double layer prototypes (squares).

\section{References}

[1] V. Peskov et al., Advances in the Development of Micropattern Gaseous Detectors with Resistive Electrodes, Nucl. Instr. Meth. A 661 (2012) 153.

[2] J. M. Bidault et al., A Novel UV Photon Detector with Resistive Electrodes, Nucl. Phys. B Proc. Suppl. 158 (2006) 199.

[3] G. Bencivenni et al., The micro-Resistive WELL: a compact spark-protected single amplification-stage MPGD, JINST 10 (2015) P02008.

[4] F. Sauli, GEM: A new concept for electron amplification in gas detectors, A 386 (1997) 531.

[5] A. Ochi et al., Carbon sputtering Technology for MPDG detectors, Proceeding of Science (TIPP2014) 351.

[6] M. Alfonsi et al., Activity of CERN and LNF groups on large area GEM detectors, Nucl. Instr. Meth. A 617 (2010) 151.

[7] M. Dixit et al., Position sensing from charge dispersion in micro-pattern gas detectors with a resistive anode, Nucl. Instr. Meth. A 518 (2004) 721.

[8] M. Raymond et al., The APV25 0.25 m CMOS readout chip for the CMS tracker, IEEE Nucl. Sci. Symp. Conf. Rec. 2 (2000) 9/113.

[9] P. Aspell et al., VFAT2: A front-end system on chip providing fast trigger information, digitized data storage and formatting for the charge sensitive readout of multi-channel silicon and gas particle detectors, URL:https://cds.cern.ch/record/1069906

[10] J. A. Merlin, Etude de fonctionnement á long terme de détecteur gazeux l'environment á haut flux de CMS, PhD Thesis (2016).

[11] G. Bencivenni et al., Performance of a triple-GEM detector for high rate charged particle triggering, Nucl. Instr. Meth. A 494 (2002) 156. 